\title{
IMPORTANT SYMPTOMS IN RELATION TO DIAGNOSIS AND TREATMENT OF MENTAL DISORDER.*
}

\author{
By W. LINDESAY NEUSTATTER, B.Sc., M.B., M.R.C.P.
}

(Assistant Physician in Psychological Medicine to Queen Mary's Hospital for the East End; Clinical Research Assistant to the Dept. of Psychological Medicine, Guy's Hospital; late Out-Patient Medical Officer to the Maudsley Hospital.)

In dealing with a case of psychological disorder, certain questions present themselves from a diagnostic point of view, viz.:

(I) Are the symptoms complained of an expression of (a) some general medical disorder, e.g., is præcordial pain due to heart disease, $(b)$ or organic disorder of the nervous system and brain?

(2) Are the symptoms an expression of psychoneurosis, a psychosis, of mental defect?

(3) What causal factors, organic or psychogenic, appear to be present?

Mental defect is a fairly clear-cut syndrome, and will be considered at the end. Of the psychoses two things need to be said. Some authorities hold there is no clear-cut line between psychosis and neurosis. Others disagree. However, for practical purposes the question is whether a given disorder is a neurosis in the sense that even if severe it will not disintegrate the patient's personality, or require certification; or is there evidence of a disintegrating process, i.e., a psychosis, which may necessitate certification?

In this paper I propose to deal chiefly with the second and third questions. The first part of Question I concerns the general consultant. As regards neurological disorders, these can broadly be subdivided into the nervous disorders which primarily affect the cord, e.g., disseminated sclerosis or subacute combined degeneration, and those which essentially affect the brain. The latter enter most into the psychiatrist's province; the former are conditions which, if present, are essentially the concern of the neurologist.

The presence of psychological symptoms appears to have the awe-inspiring effect of entering an uncharted sea, where sprats are regarded as potential monsters of the deep, yet ugly sharks pass by unrecognised. It might therefore be useful to point out that while certain symptoms are suggestive of an organic nervous disorder, others are almost certainly functional.

Somatic Symptoms. Disturbances in motor or sensory systems or of incoordination may be either organic or functional. Thus, pains, subjective sensations of numbness, of pricking feelings in the limbs, or unsteadiness, clumsiness, paralyses, difficulty in seeing, etc., all may be signs of organic nervous disorder. Opposed to these are disturbances of intellect or emotions (vide infra) and vague generalised symptoms, e.g., feelings of fullness.

A word may be said of pain in the head. When a patient complains of a "tight feeling," or a "feeling of pressure in the head," of generalised throbbing, or a tender spot at the vertex, these are almost certainly functional, and associated with depression or anxiety, and not due to a condition of the brain or skull, as patients frequently believe them to be. A full examination must of course be made to exclude organic disease, special attention being paid to the discs in case there is

-A paper read at the post-graduate course at Queen Mary's Hospital for the East End. 
tumour or renal disease. Sixth-nerve palsies should arouse suspicion as an early indication of tumour. Signs may be late. I remember a case of headache without nausea which lasted for two years, before nystagmus and slightly blurred discs heralded the presence of a tumour.

Psychological Symptoms. Changes in intelligence may be suggestive of organic disease of the brain (this is dealt with below), but feelings of anxiety or obsessional ideas are practically never due to organic disorders, as appears frequently to be thought.

Turning to the differentiation of neurosis from psychosis, it should be emphasised that as there is a gradual transition of symptoms, any symptom-however suggestive of nothing worse than a mild neurosis-may be the precursor of a psychosis, as defined. But bearing this possibility in mind, what symptoms make psychosis a probability rather than a possibility?

The symptoms which a patient may present are various. They can broadly be classified as follows: (a) Anxiety symptoms, which may be described in somatic terms, $(b)$ obsessional-compulsive symptoms, $(c)$ fatigue, and lack of concentration or impaired memory, $(e)$ changes in mood-depression or elation, $(f)$ " unreality" feelings, $(g)$ loss of certain functions, e.g., the use of the voice, a limb, etc.

All these symptoms occur in the main neuroses, anxiety states, obsessionalcompulsive states, or hysteria. How are these to be distinguished from each other, and from incipient psychoses? They can be distinguished with the aid of the history, of observations made by relatives, and in the observations made by the examiner. There may be noticed:

(I) Changes in mood, depression with or without retardation, or elation with possible "flights of ideas."

(2) Changes in personality.

(3) Intellectual changes, i.e., an inability to perform tasks compatible with what is expected of the patient from his education and his past history, or there may be tortuous and disjointed thought processes, or an inability to attend.

Taking some of these signs and symptoms:

Symptoms of a Bodily Nature. As stated, these need exclusion of organic nervous disorder, but where there are no physical signs the nature of the symptoms may help in the diagnosis. Thus, anxiety may express itself in somatic terms, which may be described as " sinking sensations," "pain and beating of the heart," "tight feelings in the head," "that my limbs will give under me," or as "passivity" feelings, e.g., as of the limbs feeling as though they were being moved. Sensations described in bizarre terms, e.g., " a creepy sensation in the ear as though a caterpillar were crawling around," should be viewed with suspicion, as suggesting schizophrenia. They should not be confused with the feelings of tingling, "as though something were crawling under the skin," which elderly patients not infrequently complain of, and which are extremely distressing but not ominous.

Anxiety states are in all probability straightforward neurosis. As already mentioned, anxiety may be expressed in bodily terms, or the patient may have specific fears, e.g., claustrophobia, agarophobia. An inability to travel in trains is a very common symptom. One of the most curious I have come across was a terror of rain, or of the threat of it, a particularly unhappy symptom for one domeciled in England. 
Only in cases where there are other observed abnormalities of the personality need a graver view be taken, and the diagnosis is made on these, and not on the anxiety symptoms.

Obsessional ideas are thoughts which enter the subject's mind against his will, and they are generally of a painful nature. A man fears he will injure his wife, a woman that she will injure her baby, or the patient cannot dismiss the thought of some past real or imaginary misdeed from his mind. Although the ideas are perpetually present and of an incongruous nature, the patient realizes that there is something out of order, and is very pained by it. If a patient begins to believe in the reality of the obsessional idea, or believe that his discomfort is being brought about by an outside agency, a paranoid illness is impending. Obsessions frequently occur in severe depressive states (melancholia), where the depression is the main feature requiring treatment. This should not be confused with a certain degree of depression which is bound to accompany such a distressing condition.

The treatment of obsessions is difficult, and with rare exceptions (e.g., hypothyroidism) requires some kind of psychotherapy. They are rarely suggestible, though I have known an elderly woman respond to hypnosis. Generally lengthy treatment of an analytical nature is needed.

Changes in Mood. Depression is one of the commonest symptoms complained of. At one extreme this is obviously " psychotic," the patient is retarded, 1.e., he drags his words out slowly and moves slowly, or he is paranoid or selfreproachful, i.e., he attributes his depression to the machinations of others or to some past transgression-however small-and he cannot be talked out of his belief. situation.

At the other extreme the depression is a natural reaction to a difficult

In between these there comes a group of conditions where the patient is depressed for no apparent reason. An example is the depression seen after childbirth, where for months afterwards there is severe listlessness, depression, and emotional instability. I think that there is a large physical component-especially fatigue in the poor. On this soil psychological difficulties flourish, especially where the mother's attitude towards the marriage or childbirth or sex, is abnormal.

"Psychotic" depressions are grave suicidal risks, and need institutional treatment. The other cases are a grave responsibility, but neurotic depression is one of the easier disorders to treat. The milder psychoses need rest, sedatives, and should on no account be encouraged to go about their daily tasks. The other depressions definitely require psychotherapy, where they can have the opportunity to talk of their troubles and to abreact. Having got their worries "off their chest," they are then malleable and open to reassurance and comfort. Many of these should be encouraged to work, but in the out-patient class, where fatigue may be a factor, the necessity for securing rest combined with psychotherapy is a serious problem. There is a terrible lack of facilities.

Elation is a tendency to overact. It is not a symptom of which the patient complains, but his over-activity and loquacity-possibly accompanied by pugnacity-may be a source of concern to the relatives. It is indicative of a hypomaniac condition, or, if severe, of mania. A different form of over-activity in the form of outbursts of excitement may also occur in schizophrenia or G.P.I. This must be distinguished from the agitation of a depressed patient. Institutional treatment is called for, but if the patient is devoid of insight it may be difficult to persuade him, while certification may always not be possible. 
Fatigue is not suggestive of psychosis, but it may be an expression of depression or anxiety. It must be differentiated from a gradual fading of interest $c$ or lack of concentration, which is a symptom which needs to be taken seriously. It may be the perfectly simple outcome of anxiety, worry, or pre-occupation with some problems. These problems may not at first be obvious. They may be deep and ramifying, and need some time to elucidate, but with their solution the inability to concentrate (which is really a shifting of attention from without to within) is restored.

In young adults lack of concentration may be an early sign of "simple" schizophrenia, and is prognostically ominous. In such a case it is generally accompanied by a great falling off in intellectual ability. Thus, the bright scholar begins to fail, and with this condition an emotional shallowness occurs, which does not occur with the neurotic. It is difficult to describe, but the observer feels a kind of wall between the patient and himself. Often there is an accompanying tendency for rather tortuous thought processes. Hypochondriacal ideas and suspicion are also frequent. Patients may be aware of their deterioration and very worrried by it. In view of the possibilities of the modern treatment of schizophrenia by cardiazol, tirazol, and insulin, apart from psychotherapeutic measures, it is extremely important to recognize the early stages of the disease, as an insidious falling off in attention is often mistaken and treated as nothing more serious than laziness.

Under the heading of failing concentration intellectual deterioration can be included. Here there is an inability to concentrate in that the patient formulates $\vec{c}$ his ideas slowly, forgets the context of what he says, and is inaccurate in his memory. It is a sign of organic brain disease such as occurs in G.P.I., senilityo arteriosclerosis, and cerebral - especially frontal - tumour. Intellectual deterioration (in hysteria the patient may mimic this symptom) is never a symptom occurring in a psychoneurosis.

Unreality feelings are feelings in which the patient complains that his surroundings appear "unreal". He feels detached and conscious of himself all the time. These feelings can occur in any severe neurosis, especially bad anxiety states. The patient is very alarmed about them, and should be emphatically reassured that they are not precursors of impending insanity.

Loss of Function. Except for organic disorder, loss of function or disordered function-whether sensory or motor-is hysterical.

Special mention might be made of loss of voice and loss of memory. The former is usually hysterical, and generally a psychological precipitant is easily elicited. As is may be associated with an unsatisfactory home, the difficulty in curing the condition is partly a problem of what can be done environmentally.

Loss of memory may be hysterical, but fugues, in which the patient is found wandering, are not uncommonly schizophrenic, and occur in organic brain disorder. The diagnosis must be made on the accompanying personality, and may at times be very difficult, as " fragmentation" of the mind, very analogous to schizophrenia, is seen in hysteria.

Mental Defect. There is only space to deal with one of the various problems which arise, that is the differentiation of the child is failing on account of emotional difficulties from the true intellectual defective. The problem is complicated because the two may coexist. 
Broadly speaking the inhibited child's answers are quantitatively poor, i.e., they are lacking. But if its confidence is won then the quality is all right. The defective's answers are qualitatively poor. They reply beside the point. A child who is asked: "What is wrong with that drawing," and is shown a woman without arms, and replies: "She's got toothache," is surely a defective-or a very subtle humorist! Intelligence tests are well worth using in assessing defect, if for no other reason than that the use of standardized questions assists the examiner in coming to a conclusion I would stress that, in the hands of those who know how to use them, intelligence tests are very useful in assessing borderline defect.

The foregoing is by no means an exhaustive list, but is a brief attempt to describe the "weighting" needed to assess the importance of different symptoms in psychological disorders.

The treatment of the various disorders requires a paper to itself. All that can be given here are some rough guides.

Institutional treatment is obviously required where the patient is dangerous to himself or others. The former is the greater risk. All depressions are potential suicidal risks. Nevertheless, there are many mild depressions where the risk is small, and the importance of keeping the patient at work is greater. Institutionalization is by no means good for all patients, and should be confined to unmanageable cases or where the home environment appears detrimental. With mild schizophrenics it is an important task for the physician to keep them going, and to give them suitable psychotherapy. The problem now arises of weighing the balance between keeping schizophrenics out of hospital or treating them by one of the "shock" methods.

The recognition of early G.P.I. is very important, as malaria is most effective in the first six months.

In conclusion, the importance of eliciting the psychological ætiology of the various disorders needs to be emphasized. In some cases the history may prove arid, and may appear irrelevant. In a good number of cases not more than an occasional vertebral spine of the psychological skeleton will be apprehended at the first interview, but in many other cases a good deal of the ætiology will be apparent. If nothing more can be attempted, at least the perception of the problem by the physician will serve as a valuable rapport with the patient. In out-patients, unfortunately, there is little time for anything else, unless there are special facilities for psychotherapy.

In this connection I would like to end by saying that deeper psychotherapy is required in a good many cases to elucidate and set right the cause of the trouble. It is hard to lay down general rules as to when it is indicated, but as a rough guide I would say that mild character difficulties in young people are very responsive. The "neurotic" depressions are good material to treat, and mild obsessionals and anxiety states may prove so. Severe obsessionals and phobias are very hard to treat. Sexual perversions can prove very difficult, but in some cases may be greatly helped. 